\title{
Multidimensional and inhomogeneity effects on scattering polarization in solar prominences
}

\author{
Ivan Milić ${ }^{1,2}$ and Marianne Faurobert ${ }^{1}$ \\ ${ }^{1}$ UMR 7293 J.L. Lagrange Laboratory, Université de Nice Sophia Antipolis, CNRS, \\ Observatoire de la Côte d'Azur, Campus Valrose, 06108 Nice, France \\ email: milic@aob.rs \\ ${ }^{2}$ Astronomical observatory Belgrade, Volgina 7, 11060 Belgrade, Serbia \\ email: marianne.faurobert@oca.eu
}

\begin{abstract}
Measurements of magnetic fields in solar prominences via Hanle effect usually assume either single scattering approximation or simple, one-dimensional, slab model in order to perform an inversion and find the unknown magnitude and the orientation of the magnetic field from spectropolarimetric observations. Here we perform self-consistent NLTE modeling of scattering polarization in inhomogeneous 2D slab, illuminated from its sides by the solar continuum radiation. We show that even in the absence of a magnetic field, in the non-optically thin regime, significant non-zero Stokes U is to be expected. Neglecting these effects, in principle, could cause systematic errors in spectropolarimetric inversions, in the case when the prominence is optically thick.
\end{abstract}

Keywords. line formation, scattering polarization, radiative transfer

\section{Introduction}

In order to measure magnetic fields pervading solar prominences one usually performs spectropolarimetric inversion. One of the most common choices for such procedure is He1083 line as demonstrated by, for example, Asensio Ramos et al. (2008). To find the unknown magnitude and the orientation of the magnetic field, one usually assumes that the emergent spectral line is formed by single scattering (optically thin regime) of the incident continuum radiation, or, in a bit more sophisticated approach, that the prominence behaves as a vertical, one-dimensional slab in which polarized radiative transfer takes place. In both of these approaches, in the absence of a magnetic field, the emergent Stokes $U$ is zero. Thus, non-zero Stokes $U$ implies the presence of a magnetic field and the ratio between Stokes $U$ and $Q$ gives information on the orientation of the field. In this paper the positive reference direction for Stokes $Q$ is parallel to solar limb.

However, prominences are sometimes optically thick in diagnostically important lines and they are far from one-dimensional. It is our assumption that a more complex and realistic prominence model would create additional polarization signatures. Here we investigate scattering polarization in a hypothetical line, formed by scattering in a twodimensional, inhomogeneous slab, in the presence of velocity fields.

\section{Prominence model and method of solution}

We consider a 2D slab, with arbitrary opacity distribution in the $x, y$ plane and homogeneous along $z$ ( $z$ is the atmospheric normal). The slab is placed 20 "above the solar surface and illuminated by the limb-darkened solar radiation where the limb darkening 

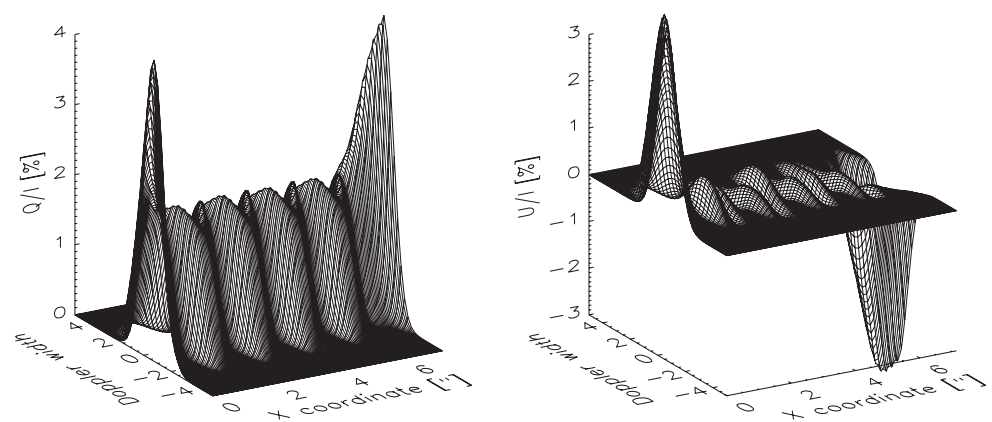

Figure 1. Spatial distribution of line profiles in the simple prominence model. Line of sight is parallel to $y$ axis, that is, corresponds to the prominence seen exactly on the limb.

coefficients correspond to the wavelength of $\approx 1100 \mathrm{~nm}$. The slab is $1000 \mathrm{~km}$ "thick" and $5000 \mathrm{~km}$ "wide". In order to emulate vertical threads, we adopt the following distribution of the line-integrated opacity:

$$
\chi(x, y)=\chi_{0} \sum_{i}^{9} \exp \left(-\left(x-x_{0, i}\right)^{2} / \sigma^{2}\right) \exp \left(-\left(y-y_{0, i}\right)^{2} / \sigma^{2}\right) .
$$

Here $\sigma=150 \mathrm{~km}$ and $\chi_{0}$ is chosen so that the mean, line-integrated, prominence optical thickness along $x$ is equal to 5 . Spacing in $x$ between the peaks of the opacity distribution is $500 \mathrm{~km}$. The plasma is also pervaded by a velocity field which has sinusoidal variations along $x$ and has two components:

$$
v_{y, z}=v_{\max } \sin \left((x-500 \mathrm{~km}) \frac{2 \pi}{1000 \mathrm{~km}}\right),
$$

where $v_{\max }$ is equal to one Doppler velocity, which, for He1083 line, corresponds to about $10 \mathrm{~km} / \mathrm{s}$.

To self-consistently solve the NLTE polarized radiative transfer problem for a two-level atom and compute the emergent Stokes vector $(I, Q, U)$, we use the reduced intensity basis as shown by Anusha \& Nagendra(2011). The formal solution is based on short characteristics approach by Olson \& Kunasz(1988) while the self-consistent NLTE solution follows the approach given by Milić(2013). We neglect collisional depolarization and use $W_{2}=0.3$ for intrinsic line polarizability. This is similar to the red wing of He1083, which consists of two lines, with $W_{2}=0.25$ and 0.35 .

\section{Results and the discusion}

Fig. 1 shows that, even in the absence of a magnetic field, the $Q / I$ and $U / I$ polarization profiles have complex shapes which vary strongly with the line of sight. Note that the spatial distribution has been smeared with a 0.5 "-wide mean filter in order to account for the finite resolving power of today's ground based instruments. It is important to notice that Stokes $Q$ is enhanced near the edges of the prominence due to the multidimensional radiative transfer effects. Stokes $U$ is also non-zero all over the prominence, again with strong peaks near the edges due to the additional anisotropy.

These simple, "toy-model" results show that, in complex prominence models there are much more effects which contribute to emergent scattering polarization then just the Hanle effect acting on the scattering of the anisotropic incident radiation. In subsequent 
work we will explore the interplay between these effects and the Hanle effect resulting from large scale magnetic field pervading the prominence.

\section{References}

Anusha, L. S. \& Nagendra, K. N. 2011, ApJ, 726, 6

Asensio Ramos, A., Trujillo Bueno, J., \& Landi Degl'Innocenti, E. 2006, ApJ, 683, 542 Milić, I. 2013, $A \& A A, 555 \mathrm{~A}, 130 \mathrm{M}$

Kunasz, P. B. \& Olson, G. L. 1988, JQSRT, 39, 1 